\title{
Cornea and Refractive Atlas of Clinical Wisdom Eds: S. A. Melki and M. A. Fava 2011 272pp., Hardcover ISBN: 978-1-55642-867-8 Slack Inc
}

\author{
Adrian Tey $\cdot$ Stephen B. Kaye
}

Received: 23 August 2011 /Accepted: 25 August 2011 /Published online: 8 September 2011

(C) Springer-Verlag 2011

The hard-cover book 'Cornea and Refractive Atlas of Clinical Wisdom' is edited by SA Melki and MA Fava, was first published in March 2011 by Slack Inc. The book assembles the collective wisdom and knowledge of a number of experienced surgeons who are experts in their chosen field.

The book has 249 pages divided into seven sections and 32 chapters. These sections consist of 'Imaging and Diagnostics', 'Ocular Surface Disorders', 'Infectious Diseases', 'Inflammatory Diseases', 'Neoplasia and Epithelial Downgrowth', 'Dystrophies and Degenerations', and finally 'Refractive Surgery'. Within each section, each chapter begins with a detailed description of the condition from diagnosis to symptoms to complications and to eventual treatment methods. One particular feature, which is of interest and without a doubt helpful, is the 'In My Experience' section where individual authors share their personal experience and diagnostic pearls on their particular subject. On certain subjects, it is interesting to find that much of their anecdotal experiences have been well-founded over the years compared to the type of information that is awaited from a large clinical trial. Other good features of the book include emphasis on the provision of practical tips, with less dependence on theoretical aspects of particular diseases. The authors are all experts and well-published in the areas each has covered within the book.

Overall, this book is well-structured and easy to digest. The accompanying illustrations are of good standard, as expected in an 'atlas'. The editors have done well in gathering all the authors to share their experience collected over years of clinical practice. This book will be extremely useful for trainees, general ophthalmologists and even experienced corneal and refractive surgeons as a textbook or quick reference guide for practical tips on management.

A. Tey $\cdot$ S. B. Kaye $(\bowtie)$

St. Paul's Eye Unit, Ophthalmology, Royal Liverpool University Hospital, 8Z Link, Prescot Street, Liverpool, Merseyside L7 8XP, UK

e-mail: s.b.kaye@liverpool.ac.uk 